\title{
Qualidade de vida entre residentes multiprofissionais em saúde
}

\section{Quality of life among multiprofessional residents in health}

\begin{abstract}
Graziela Lopes de Oliveira Hospital Universitário da Universidade Estadual de Ponta Grossa - HU-UEPG - Ponta Grossa - Brasil glopes.olv@gmail.com

Danielle Bordin Universidade Estadual de Ponta Grossa - UEPG - Ponta Grossa - Brasil daniellebordin@hotmail.com

Cristina Berger Fadel Universidade Estadual de Ponta Grossa - UEPG - Ponta Grossa - Brasil cbfadel@gmail.com
\end{abstract}

Luciane Patrícia Andreani Cabral Hospital Universitário da Universidade Estadual de Ponta Grossa - HU-UEPG - Ponta Grossa - Brasil luciane.pacabral@gmail.com

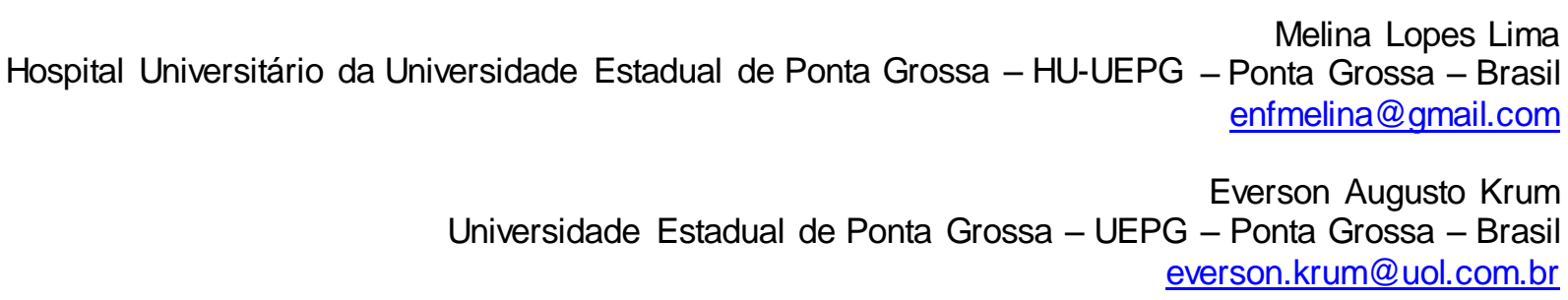

\section{Resumo}

As residências multiprofissionais em saúde foram criadas como estratégia para melhorar o processo de formação dos profissionais em saúde a fim de alcançar o nível desejado de qualidade na assistência e na atenção a saúde ofertados pelo Sistema Único de Saúde. A ciência que a qualidade de vida (QV) de um indivíduo pode interferir na sua produtividade e qualidade do trabalho, torna relevante a discussão sobre seu impacto na vida dos profissionais residentes em saúde. Neste sentido, o presente estudo objetiva analisar a qualidade de vida de residentes multiprofissionais em saúde de um hospital universitário, tendo como referência os domínios físico, social, psicológico e ambiental. Trata-se de um estudo transversal do tipo inquérito, quantitativo, realizado com a totalidade de residentes ingressantes e concluintes de um hospital universitário no ano de 2018, utilizando o questionário WHOQOL-BREF. Em todos os domínios investigados os escores de QV de ambos os grupos foram semelhantes $(p>0,05)$, apresentando maior escore os domínios físico e das relações sociais e menor escore o do meio ambiente. A análise das condições demográficas versus domínios, encontrou diferença significativa no domínio psicológico para a variável idade $(p=0.015)$, quando comparado residentes com até 26 anos (média 64.84) e maiores de 26 anos (média de 73.61). O presente estudo possibilitou analisar e conhecer os pontos de fragilidade e de potencialidade na QV de 
profissionais residentes e fomentou questões relevantes para a compreensão do contexto do trabalho do residente multiprofissional.

Palavras-chave: qualidade de vida, formação profissional em saúde, especialização, serviços de saúde, atenção terciária à saúde.

\begin{abstract}
Multiprofessional residencies in health were developed as a strategy to improve the training process of health professionals for the purpose of achieving the desired quality level of care and health care offered by the Public Health System. The acknowledgment of a person quality of life (QoL) can interfere with their productivity and quality of work, makes relevant the discussion about its impact on the lives of health professional residents. Therefore the present study aims to analyze the quality of life of multiprofessional health residents of a university hospital, taking as reference the physical, social, psychological and environmental domains. This is a cross-sectional, quantitative study, developed with all the first and last year residents of a university hospital in 2018, using the WHOQOLBREF questionnaire. In all domains investigated, the QoL scores of both groups were similar ( $p>0.05$ ), with higher physical and social relationships and lower environmental scores. The analysis of demographic conditions versus domains found a significant difference in the psychological domain for the age variable $(p=0.015)$ when compared to residents up to 26 years old (average 64.84) and over 26 years old (average 73.61). The present study made it possible to analyze and know the points of fragility and potentiality in the QoL of professional residents and raised relevant questions for the understanding in work context of the multiprofessional resident.
\end{abstract}

Keywords: quality of life, health human resource training, specialization, health services, tertiary healthcare.

\title{
1. Introdução
}

No Brasil, a Constituição Federal oficializou o processo de criação de um sistema de saúde público, universal e descentralizado, o Sistema único de Saúde (SUS), o qual infere sobre políticas públicas de âmbito municipal, regional e federal (BRASIL, 1988). Desde a sua instituição, o SUS vem enfrentando mudanças organizacionais para atingir um nível desejado de qualidade na assistência e atenção à saúde, visando reestruturar o modelo de saúde brasileiro. No entanto, de acordo com o Ministério da Saúde (MS) e com a Secretaria de Gestão do Trabalho e da Educação na Saúde (SGTES), é necessário que existam também mudanças na formação dos profissionais da saúde, com vistas ao incremento e ao aprimoramento da qualidade no atendimento à saúde da população (FERNANDES, 2013).

Com o desenvolvimento tecnológico e sociocultural, associados à globalização no mercado de trabalho, as exigências de responsabilidade, produtividade, iniciativa, habilidades e excelência no desempenho das funções também estão aumentando. $\mathrm{Na}$ área da saúde, as diretrizes e os princípios do SUS são o referencial teórico e, por vezes, metodológico, para as competências e habilidades que os profissionais da saúde necessitam adquirir. Novas políticas e programas são criados constantemente a fim de que trabalhadores apreendam, em sua formação, conceitos de compromisso e corresponsabilização na produção de saúde, o estabelecimento de vínculos solidários e de participação coletiva no processo de gestão, a identificação das necessidades de saúde da população e a mudança nos modelos de atenção e gestão dos processos de trabalho (BRASIL, 2007; GOULART et al., 2012).

Nessa perspectiva, surge em 2005, a modalidade de formação especializada em serviço nomeada Residência Multiprofissional em Saúde (RMS), a qual representa uma 
das estratégias potenciais para o repensar do processo de formação em saúde e das mudanças necessárias na produção de saúde no Brasil, por possibilitar o contato profissional simultâneo com o mundo do trabalho e da formação educacional (CECCIM, 2010; SILVA et al., 2015), em um universo que privilegia a atuação em equipe interdisciplinar e multiprofissional.

Vinculando então o binômio formativo trabalho e ensino multiprofissional, o qual integra diferentes núcleos de saberes, torna-se relevante a discussão sobre o seu impacto na qualidade de vida (QV) de profissionais residentes em saúde, tendo em vista o reconhecimento de fatores que possam ser efetivamente transformados pela implementação de políticas de gestão. Apesar de não haver consenso sobre a conceituação de qualidade de vida, ela é aceita como a percepção do indivíduo em relação a sua posição na vida no contexto da cultura e sistema de valores nos quais ele vive e em relação aos seus objetivos, expectativas, padrões e preocupações (WHO, 1996), frequentemente relacionada a condições ambientais, família, saúde, cultura, lazer, educação, políticas governamentais, o próprio indivíduo e o trabalho (KILIMNIK; MORAES, 2000). Sendo então a QV fenômeno subjetivo e multidimensional, a satisfação e o bem-estar do indivíduo na execução de suas tarefas podem interferir na produtividade e na qualidade do trabalho em saúde, em especial, de profissionais que atuam nessa modalidade recente de residência em saúde, a RMS.

Nesse sentido, o estudo objetiva analisar a qualidade de vida de residentes multiprofissionais em saúde de um hospital universitário, tendo como referência os domínios físico, social, psicológico e ambiental.

\section{Método}

O presente estudo foi aprovado pelo Comitê de Ética em Pesquisas com seres humanos de uma instituição de ensino (parecer no 2.461.494/2018), respeitando os ditames da resolução 466/12 do Conselho Nacional de Saúde.

Configura-se em um estudo transversal, tipo inquérito, quantitativo, realizado com a totalidade de residentes ingressantes $(n=61)$ e concluintes $(n=37)$ dos programas de residência multiprofissional em saúde de um hospital universitário (ano base: 2018). A perda da amostra ocorreu devido a não aceitação em participar do estudo $(n=22)$.

A coleta de dados foi realizada por pesquisador treinado, com vistas a explanar o objeto da pesquisa, seu caráter de voluntariedade e de não-identificação, assim como sobre a forma de coleta, análise e destino dos dados. Os que aquiesceram com sua participação, o fizeram, inicialmente mediante o preenchimento de um Termo de Consentimento Livre e Esclarecido e em seguida responderam ao questionário autoaplicável. Os dados foram coletados de forma coletiva, em sala de aula.

O questionário utilizado foi WHOQOL-BREF, versão abreviada em português do Instrumento de Avaliação de Qualidade de Vida da Organização Mundial de Saúde, valido por Fleck et al. (2000). Esse instrumento contém 26 questões distribuídas em quatro domínios: relações sociais, psicológico, físico e meio ambiente. Cada domínio é composto por questões cujas pontuações das respostas variam na escala de Likert de 1 e 5.

Os dados foram tratados de acordo com o preconizado pela Organização Mundial da Saúde, detalhado por Pedroso et al. (2010), para execução em Microsoft Excel, o qual agrupa as variáveis nos 4 domínios e calcula a média destes agrupamentos e ainda expõe a média de avaliação global de qualidade de vida.

Considerou-se como variável dependente os domínios do WHOQOL-BREF e a avaliação global de qualidade de vida, e como variáveis independentes, a posição do residente no programa de pós-graduação, ingressantes ou concluintes e as características demográficas (sexo, idade e estado civil). 
Para análise empregou-se o teste de normalidade (Kolmogorov-Smirnov) a fim de definir a utilização de análise paramétrica ou não paramétrica. Todos os dados apresentaram distribuição normal, deste modo, aplicou-se o teste paramétrico tde Student não pareado para a comparação das médias dos domínios segundo status no hospital e características demográficas. Considerou-se o nível de significância de $5 \%$ e o programa utilizado para realização dos testes foi o GraphPadlnSat (GraphPad Software, San Diego, Califórnia, USA, www.graphpad.com).

\section{Resultados}

Os resultados indicaram a participação de 98 residentes, de um universo de 120 , com 18,3\% de perdas, sendo 61 ingressantes e 37 concluintes. A média etária dos ingressantes foi de 25,6 anos $(21 \pm 43 ; d p=4,14)$, solteiros $(n=50 ; 82 \%)$ e mulheres $(n=54$; $88 \%)$ em sua maioria. Os residentes concluintes tinham em média 26,12 anos (22 \pm 46 ; $d p=5,00)$, sendo a maioria também mulheres ( $n=32 ; 87 \%)$ e solteiros $(n=27 ; 73 \%)$.

A Figura 1 apresenta os resultados de qualidade de vida dos residentes, segundo posição na residência e dissociação em domínios. Em todos os domínios investigados os escores de qualidade de vida de ambos os grupos foram muito semelhantes, não havendo diferença significativa entre eles $(p>0,05)$. Os domínios de maior escore foram 0 físico e o das relações sociais e o de menor escore o do meio ambiente.

$\mathrm{Na}$ análise de condições demográficas versus domínios, verificou-se diferença significativa apenas no domínio psicológico, para a variável idade $(p=0.015)$, onde os indivíduos com até 26 anos apresentaram uma média de 64.84 ( $\mathrm{dp} \pm 16.12)$, significativamente menor do que os indivíduos com mais de 26 anos (73.61; dp \pm 13.96 ). Nos demais domínios e características demográficas não houve diferença significativa $(p>0,05)$ (Tabela 1).

Figura 1: Média e desvio padrão (DP) de qualidade de vida de residentes, segundo posição e domínio

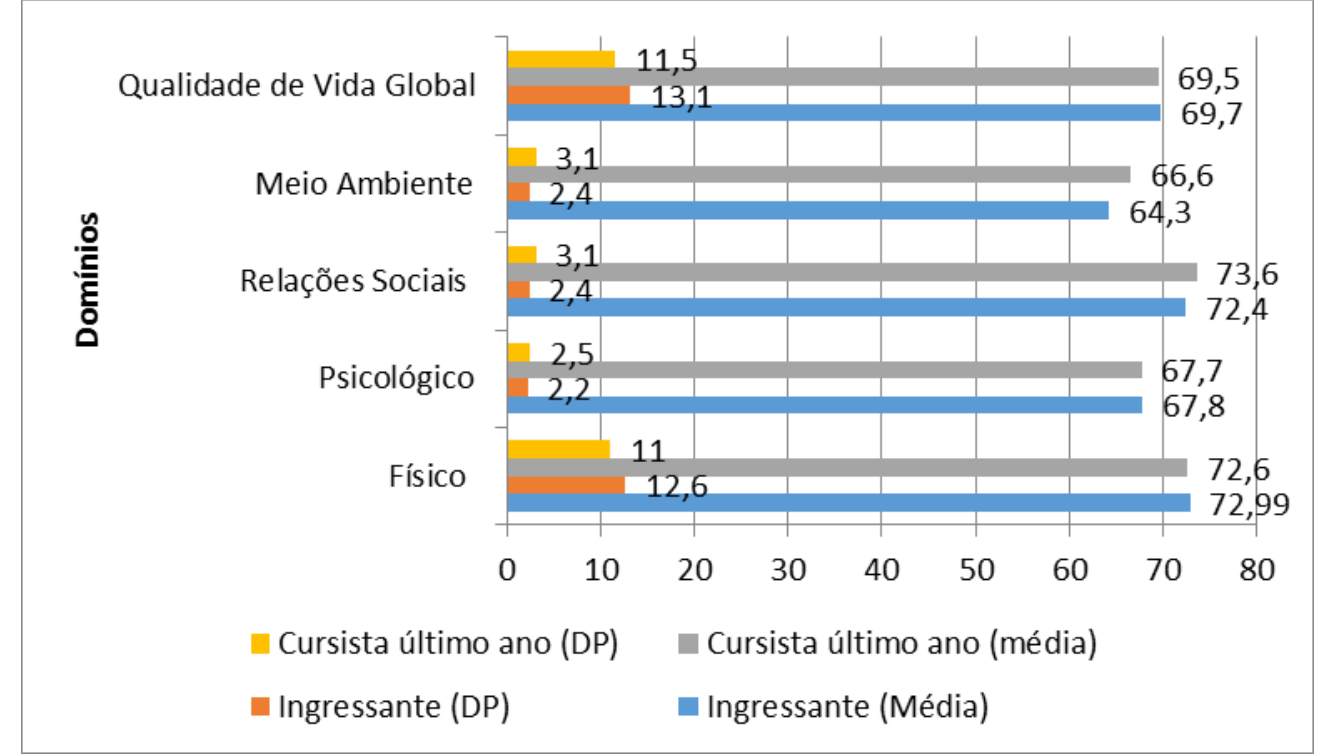

Fonte: Autores (2018)

Tabela 1: Média, desvio padrão (DP) e valor de $p$, de condições demográficas, segundo domínios de qualidade de vida de residentes multiprofissionais em saúde, 2018 ( $n=98$ ).

\begin{tabular}{ccccccccccc}
\hline $\begin{array}{c}\text { Variável } \\
\begin{array}{c}\text { Demográ- } \\
\text { fica }\end{array}\end{array}$ & $\begin{array}{c}\text { Físico } \\
\text { Média } \\
\text { (DP) }\end{array}$ & $\begin{array}{c}\mathbf{p} \\
\text { valor }\end{array}$ & $\begin{array}{c}\text { Média } \\
\text { (DP) }\end{array}$ & $\begin{array}{c}\mathbf{p} \\
\text { valor }\end{array}$ & $\begin{array}{c}\text { Média } \\
\text { (DP) }\end{array}$ & $\begin{array}{c}\mathbf{p} \\
\text { valor }\end{array}$ & $\begin{array}{c}\text { Média } \\
\text { (DP) }\end{array}$ & $\begin{array}{c}\mathbf{p} \\
\text { valor }\end{array}$ & $\begin{array}{c}\text { Média } \\
\text { (DP) }\end{array}$ & $\begin{array}{c}\mathbf{p} \\
\text { valor }\end{array}$ \\
\hline Idade & & & & & & & & & & \\
\hline $21 \leq 26$ & 71,76 & 0,06 & 64,84 & ${ }^{*} 0,01$ & 71,56 & 0,17 & 64,00 & 0,18 & 68,57 & 0,28
\end{tabular}




\begin{tabular}{|c|c|c|c|c|c|c|c|c|c|c|}
\hline $\begin{array}{c}\text { anos } \\
>26 \text { anos }\end{array}$ & $\begin{array}{c}(12,11) \\
76,85 \\
(12,30)\end{array}$ & 8 & $\begin{array}{c}(16,12) \\
73,61 \\
(13,96)\end{array}$ & 5 & $\begin{array}{c}(17,83) \\
77,24 \\
(18,04)\end{array}$ & 0 & $\begin{array}{c}(13,17) \\
67,97 \\
(12,74)\end{array}$ & 3 & $\begin{array}{c}(17,74) \\
71,63 \\
(21,95)\end{array}$ & 9 \\
\hline \multicolumn{11}{|l|}{ Sexo } \\
\hline $\begin{array}{l}\text { Feminino } \\
\text { Masculino }\end{array}$ & $\begin{array}{c}72,57 \\
(12,52) \\
77,53 \\
(10,17) \\
\end{array}$ & $\begin{array}{c}0,19 \\
3\end{array}$ & 74,65 & $\begin{array}{c}0,08 \\
9\end{array}$ & $\begin{array}{c}73,14 \\
(18,01) \\
72,92 \\
(18,51) \\
\end{array}$ & 0,97 & $\begin{array}{c}64,63 \\
(12,80) \\
68,53 \\
(15,24) \\
\end{array}$ & $\begin{array}{c}0,33 \\
7\end{array}$ & $\begin{array}{c}69,13 \\
(18,76) \\
71,59 \\
(20,98) \\
\end{array}$ & $\begin{array}{c}0,68 \\
7\end{array}$ \\
\hline \multicolumn{11}{|c|}{ Estado Civil } \\
\hline $\begin{array}{l}\text { Solteiro } \\
\text { Casado }\end{array}$ & $\begin{array}{c}73,30 \\
(12,58) \\
72,77 \\
(11,54) \\
\end{array}$ & $\begin{array}{c}0,86 \\
4\end{array}$ & $\begin{array}{c}67,15 \\
(16,21) \\
67,92 \\
(15,42) \\
\end{array}$ & $\begin{array}{c}0,84 \\
9\end{array}$ & $\begin{array}{c}72,97 \\
(18,33) \\
73,68 \\
(16,96) \\
\end{array}$ & $\begin{array}{c}0,87 \\
8\end{array}$ & $\begin{array}{c}65,87 \\
(12,81) \\
62,23 \\
(14,16) \\
\end{array}$ & $\begin{array}{c}0,27 \\
1\end{array}$ & $\begin{array}{c}69,76 \\
(18,27) \\
68,12 \\
(21,64) \\
\end{array}$ & $\begin{array}{c}0,73 \\
3\end{array}$ \\
\hline
\end{tabular}

${ }^{*} \mathrm{p}<0,05$

Fonte: Autores (2018)

\section{Discussão}

Os estudos relacionados aos residentes multiprofissionais descritos na literatura têm revelado semelhança no perfil sociodemográfico encontrado no presente estudo, com maioria absoluta de profissionais entre 20 e 30 anos (ROTTA et al., 2019; OSHIMA et al., 2018; PÉREZ; SANTOS; DÓRO, 2017; MOREIRA et al., 2016), demonstrando que os programas de residência tem sido uma opção bastante considerada pelos recémformados (OSHIMA et al., 2018; MOREIRA et al., 2016); predominância de solteiros e de mulheres (ROTTA et al., 2019; OSHIMA et al., 2018; PÉREZ; SANTOS; DÓRO, 2017; MOREIRA et al., 2016), perfil já reconhecido como uma tendência das profissões da área da saúde.

O domínio das relações sociais foi o domínio com maior média nesta pesquisa. Outros estudos encontraram resultados semelhantes (ASAIAG et al, 2010; DIAS et al, 2016). Um estudo nacional com residentes multiprofissionais atribuiu o tempo gasto com rotinas do dia a dia, como deslocamento entre moradia e trabalho como um influenciador neste domínio, diminuindo o tempo livre para as relações sociais (ROCHA; CASAROTTO; SCHMITT, 2018), portanto, acredita-se que nesse sentido, os residentes deste estudo possam ter fixado moradia próxima ao hospital, facilitando seu deslocamento, ou até que possuam locomoção própria. Além disso, acredita-se que a própria residência multiprofissional influencie nas relações sociais, uma vez que permite conhecer novas pessoas e firmar novas amizades no próprio ambiente de trabalho e estudo.

O domínio físico foi o segundo domínio identificado com maior escore no presente estudo. Este domínio também foi evidenciado com maior escore em estudos brasileiros incluindo residentes médicos (ASAIAG et al, 2010; DIAS et al, 2016). Tendo em vista que os residentes são majoritariamente jovens adultos, pode-se explicar o fato de o domínio físico ser o menos afetado durante o período de residência, uma vez que quanto maior a idade, menor a frequência de atividades físicas e maior a prevalência de doenças incapacitantes (ALMEIDA-BRASIL et al, 2017). Outro fator que parece influenciar o domínio físico é a maior disposição para realizar atividades de lazer, podendo influenciar positivamente neste domínio (ROCHA; CASAROTTO, SCHMITT, 2018).

Contrariamente, quando considerada a literatura apenas dos residentes multiprofissionais, estudos brasileiros mostram que o domínio físico é um dos mais afetados nestes residentes (CAHÚ et al, 2014; LOURENÇÃO; MOSCARDINI; SOLER, 2013; VIEIRA et al, 2018). O domínio físico é diretamente influenciado por dor física, energia para o dia a dia, sono e desempenho de atividades diárias (LOURENÇÃO; MOSCARDINI; SOLER, 2013). Acredita-se que o desgaste físico seja o responsável por este achado, pois um estudo verificou que o desgaste físico constante e cansaço excessivo, estiveram presentes em $80 \%$ da amostra de residentes multiprofissionais (CAHÚ et al, 2014). 
Nesse sentido, acredita-se que a maior média encontrada no domínio físico neste trabalho seja explicada pela média de idade baixa dos residentes do estudo (25,6 anos), diminuindo o risco de doenças incapacitantes. O cansaço físico e sono, muito discutido em outros estudos, parece não ter afetado tão negativamente este domínio neste estudo (CAHÚ et al, 2014; LOURENÇÃO; MOSCARDINI; SOLER, 2013; VIEIRA et al, 2018).

A menor média de QV encontrada neste trabalho foi no domínio ambiental, que também foi encontrada em outros estudos (ASAIAG et al, 2010; DIAS et al, 2016; ROCHA; CASAROTTO, SCHMITT, 2018). Novamente relaciona-se a disponibilidade de tempo para as atividades pessoais além do programa de residência como influenciadora do nível de satisfação. Autores encontraram que o tempo para lazer é um fator de proteção para insatisfação no domínio ambiental (ROCHA; CASAROTTO; SCHMITT, 2018).

O domínio ambiental é constituído por questões relacionadas à segurança física e proteção, recursos financeiros, condições do ambiente físico, transporte, condições de moradia, entre outras. Desse modo, além das questões relacionadas ao lazer, acredita-se que as condições de trabalho dos residentes e também de moradia tenham influenciado neste domínio. Considerando que a fase da vida em que se encontram, é possível que a maioria dos residentes esteja vivendo em moradia compartilhada, o que pode diminuir a privacidade e impactar na QV.

Além disso, o ambiente hospitalar é repleto de situações com pacientes envolvendo sofrimento, dor, acidentes, tristeza e estresse, o que pode influenciar e acarretar danos ao profissional, uma vez que o mesmo desenvolve suas atividades diárias nesses cenários, e pode ser afetado psicologicamente e fisicamente (HARBS; RODRIGUES; QUADROS, 2008) podendo impactar negativamente na QV, principalmente no domínio ambiental.

No presente estudo, a qualidade de vida de residentes multiprofissionais ingressantes e concluintes em saúde apresentou diferença significativa apenas no domínio psicológico, quando comparado residentes com até 26 anos e mais de 26 anos, sendo a QV menor no grupo de menor idade. Acredita-se que as diferentes fases da vida são marcadas por preocupações e anseios distintos, o que pode influenciar na QV.

O domínio psicológico é formado pelas seguintes facetas: imagem corporal e aparência; sentimentos positivos e negativos; autoestima; espiritualidade, religião e crenças pessoais; pensamento, aprendizagem, memória e concentração (WHO, 1996).

A melhora na QV de indivíduos mais velhos foi corroborada por um estudo espanhol, que verificou ligeiro aumento da média no domínio psicológico conforme o avançar da idade em profissionais médicos experientes quando comparados com residentes médicos mais jovens (IRARRAZÁVEL; HALPERN; HUNEEUS, 2012).

Uma explicação para a menor QV nos residentes mais jovens pode ser atribuída à maior probabilidade de este grupo etário compor em sua maioria o grupo de ingressantes e neste caso, o início da residência é marcado por altas expectativas, visto que por vezes é o primeiro emprego, decorrente das horas de estudo para o ingresso no programa, que em algumas ocasiões, ao ingressar não são supridas, podendo gerar sentimentos negativos (REZENDE et al., 2011).

Ademais, o início da carreira profissional, pela transição da posição de acadêmico para profissional, corriqueiramente é marcado por sentimentos capazes de diminuir a autoestima do profissional, como: desvalorização, insegurança, ansiedade e incompetência (MOREIRA et al., 2016). Somado a isso, as residências multiprofissionais têm uma carga horária extensa de 60 horas semanais e muitos residentes se afastam da família ou do círculo de amizades por motivo de mudança para ingressar na residência (GUIDO et al., 2012), sendo possível que esta mudança no estilo de vida, interfira na QV.

Em contrapartida, um estudo norte-americano que verificou a QV em residentes médicos apresentou uma pior média de QV em residentes maiores de 40 anos quando comparado aos mais jovens no que se refere à qualidade de vida geral, autocuidado, 
autossatisfação e relações interpessoais (KOVACH et al., 2016), atribuída a preocupações com filhos, saúde, pais idosos e vida financeira, além dos próprios estressores relacionados à residência. Um estudo brasileiro encontrou diferença no domínio físico da QV, quando comparado residentes médicos maiores e menores de 26 anos, com pior média no grupo com maior idade (MACEDO et al., 2009), inferindo que outros fatores como tempo para lazer e atividade física regular explicariam esse achado.

Uma característica relacionada com a QV é a satisfação, que é definida por como o indivíduo se sente diante de uma situação. A satisfação do residente com o programa de residência pode influenciar no modo como estes profissionais realizam suas atividades e como lidam com as dificuldades no trabalho, afetando os aspectos emocionais da QV (MACEDO et al., 2009), além disso, a satisfação pode influenciar na produtividade, interferindo diretamente no cuidado com o paciente (UMANN; GUIDO; GRAZZIANO, 2012; LOURENÇÃO; MOSCARDINI; SOLER, 2013).

A literatura consultada sobre QV de residentes multiprofissionais e médicos, nacionais e internacionais, em sua maioria, não verificam a associação entre as variáveis qualidade de vida e idade, realizando-se apenas a descrição do perfil sociodemográfico da amostra do estudo (ROCHA; CASAROTTO; SCHMITT, 2018; DIAS et al., 2016; BAHÚ et al., 2014; FERNANDEZ-PRADA et al., 2014; REZENDE et al., 2011; AISAIAG et al., 2010; SCHONHAUTet al., 2009). Nesse sentido, este estudo sugere a inclusão da associação da idade com a QV em estudos futuros com vistas a aprofundar o tema (KOVACH et al., 2016).

A limitação deste estudo decorreu da ausência de controle dos fatores de confusão da QV como: burnout, estresse, dor, renda, formação profissional, experiência de violência e setor de atuação (CAVALCANTI, 2018), uma vez que os fatores influenciadores indicados em estudos anteriores permitiriam melhor compreensão da QV.

\section{Considerações finais}

Apesar de haver diferença significativa na QV de residentes multiprofissionais ingressantes e concluintes apenas do domínio psicológico, o presente estudo possibilitou analisar e conhecer os pontos de fragilidade e de potencialidade na QV destes profissionais e fomentou questões relevantes para a compreensão do contexto do trabalho do residente multiprofissional. Acredita-se que este conhecimento possibilitará o planejamento de medidas de melhoria de QV de residentes, influenciando na vida pessoal do residente e em seu desempenho durante o período da residência, podendo afetar positivamente nos serviços de saúde e na qualidade da assistência prestada ao paciente. Dado o impacto que a QV pode representar, novos estudos que aprofundem o tema se fazem necessários.

\section{Referências}

ALMEIDA-BRASIL, C. C. et al. Qualidade de vida e características associadas: aplicação do WHOQOL-BREF no contexto da Atenção Primária à Saúde. Ciência Saúde Coletiva, v. 22 , p. $1705-1716,2017$.

ASAIAG, Paulo Eduardo et al. Avaliação da qualidade de vida, sonolência diurna e burnout em médicos residentes. Rev Bras Educ Med, v. 34, n. 3, p. 422-9, 2010.

BERLIM, M. T; FLECK, M.P. "Quality of life": a brand new concept for research and practice in psychiatry. RevBras Psiquiatr., n. 25, v. 4, 249-52, 2003.

BRASIL. Constituição da República Federativa do Brasil de 1988. Brasília, DF: 
Presidência da República, 1988.

BRASIL. Ministério da Saúde. HumanizaSUS: Gestão participativa.Co-Gestão 2. ed. Brasília: Ministério da Saúde, 2007.

CAHÚ, R. A. G. et al. Estresse e qualidade de vida em residência multiprofissional em saúde. Rev. bras.ter. cogn., v. 10, n. 2, p. 76-83, 2014.

CAVALCANTI, I. S. et al. Burnout e depressão em residentes de um programa multiprofissional em oncologia: estudo longitudinal prospectivo. Revista Brasileira de Educação Médica, v. 42, n. 1, p. 190-8, 2018.

CECCIM, R. B. Residências em saúde: as muitas faces de uma especialização em área profissional integrada ao SUS. In: FAJARDO, A. P.; ROCHA. C. M. F.; PASSINI, V. R. (Org.). Residências em saúde: fazeres \& saberes na formação em saúde. Porto Alegre: Hospital Nossa Senhora da Conceição, 2010.

DIAS, B. A. et al. Qualidade de vida de médicos residentes de um hospital escola. Sci Med. v. 26, n. 1, p. 1-9, 2016.

FERNANDES, M. N. S. Prazer e sofrimento no processo de formação de residentes multiprofissionais de saúde. 2013, Dissertação (Mestrado em Enfermagem) Universidade Federal de Santa Maria. Santa Maria, 2013.

FERNANDEZ-PRADA, M. et al. Calidad de vida relacionada con la salud en una muestra de médicos internos residentes que realizan guardias en un Servicio de Urgencias: una perspectiva de género. Rev. méd. Chile, v. 142, n. 2, p. 193-198, 2014.

FLECK, M. P. A. et al. A aplication of the portuguese revision of the abreviated instrument of quality life. WHOQOL-bref. Rev Saúde Pública. v. 34, n. 2, p. 178-83, 2000.

GESSNER, L. S. Qualidade de vida de trabalhadores de equipes de saúde de família no sul do Brasil. Revista Brasileira de Pesquisa em Saúde, v. 15, n. 3, p. 30-37, 2013.

GOULART, T. Perfil sociodemográfico e acadêmico dos residentes multiprofissionais de uma universidade pública. Revista da Rede de Enfermagem do Nordeste, v. 13, n. 1, p. 178-186, 2012.

GUIDO, L. A. et al. Síndrome de Burnout em residentes multiprofissionais de uma universidade pública. Ver Esc Enferm USP. v. 46, n. 6, 1477-83, 2012.

HARBS, T. C.; RODRIGUES, T.; QUADROS, V. A. S. Estresse da equipe de enfermagem em um centro de urgência e emergência. Universidade Tuiti do Paraná, v. 1, n. 2, 2008.

IRARRÁZAVAL, M; HALPERN, M; HUNEEUS, Javiera. Calidad de vida en Psiquiatras Infanto-Juveniles, Neuropediatras y residentes de Psiquiatría Infanto-Juvenil: uso de laencuesta WHOQOL-BREF. Rev. Chil. Psiquiatr. Neurol. Infanc. Adolesc. v. 23, n. 1, p. 8-19, 2012. Disponível em: http://www.institutoidip.com/wpcontent/uploads/2018/11/6.pdf\#page=8. Acesso em: 31 jul. 2019.

KILIMNIK, Z. M; MORAES, L. F. R. O conteúdo significativo do trabalho como fator de qualidade de vida organizacional. Revista da Angrad., v. 1, n. 1, p. 64-74, 2000. 
KOVACH, J. G. et al. Psychiatry Resident Quality of Life. Acad Psychiatry, v. 40, n. 1, p. 76-80, 2016.

LOURENÇÃO, L. G.; MOSCARDINI, A. C.; SOLER, Z. A. S. G. Qualidade de vida de residentes não médicos. Rev. enferm UFPE, v. 7, n. 11, p. 6336-45, 2013.

MACEDO, P. C. M. et al. Health-related quality of life predictors during medical residency in a random, stratified sample of residents. Rev. Bras. Psiquiatr., v. 31, n. 2, p. 119-124, 2009.

MOREIRA, A. P. F. et al. Avaliação da qualidade de vida, sono e Síndrome de Burnout dos residentes de um programa de residência multiprofissional em saúde. Medicina, Ribeirão Preto. Online, v.49, n. 5, p. 393-402, 2016.

OSHIMA, A. et al. Perfil, atuação e satisfação de cirurgiões-dentistas em Residências Multiprofissionais em Saúde da região Sul do Brasil. Revista da ABENO. v. 18, n. 1, p. 134-45, 2018.

PEDROSO, B. et al. Cálculo dos escores e estatística descritiva do WHOQOL-bref através do Microsoft Excel. Revista brasileira de qualidade de vida. v. 2, n. 1, p. 31-6, 2010.

REZENDE, G. L. et al. A qualidade de vida entre os residentes de Otorrinolaringologia do Distrito Federal. Braz. j. otorhinolaryngol. v. 77, n. 4, p. 466-472, 2011.

ROCHA, J. S; CASAROTTO, R. A; SCHMITT, A. C. B. Saúde e trabalho de residentes multiprofissionais. Rev. Cienc. Salud, v. 16, n. 3, p. 447-462, 2018.

ROTTA, D. S. et al. Engagement de residentes multiprofissionais em saúde. Rev. esc. enferm. USP, v. 53, e03437, p. 1-8, 2019.

SCHONHAUT, B. et al. Calidad de vida de los médicos residentes del Programa de Formación de Especialistas en Pediatría. Rev. chil. pediatr., v. 80, n. 1, p. 30-38, 2009.

SILVA, S. A. M. et al. Residencia en políticas públicas: una experiencia innovadora de formación. Cad. Pesqui. v. 45, n. 157, p.588-611, 2015.

UMANN, J; GUIDO, L. A; GRAZZIANO, E. S. Presenteeism in hospital nurses. Revista Latino-Americana de Enfermagem, v. 20, n. 11, p. 159-66, 2012.

WHO. World Health Organization. Programme on mental health. WHOQOL-BREF, introduction, administration, scoring and generic version of the assessment. Geneva, 1996. 